\title{
Genetic polymorphisms identify in species/ biovars of Brucella isolated in China between 1953 and 2013 by MLST
}

Dong-ri Piao ${ }^{1 \dagger}, \mathrm{Xi} \mathrm{Liu}^{1 \dagger}$, Dong-dong Di ${ }^{2 \dagger}, \mathrm{Pei} \mathrm{Xiao}^{3+}$, Zhong-zhi Zhao ${ }^{4}$, Li-qing Xu', Guo-zhong Tian', Hong-yan Zhao', Wei-xing Fan' ${ }^{2}$, Bu-yun Cui ${ }^{1}$ and Hai Jiang ${ }^{1 *}$ (i)

\begin{abstract}
Background: Brucellosis incidence in China is divided into three stages: high incidence (1950s-1960s), decline (1970s-1980s), and re-emergence (1990s-2010s). At the re-emergence stage, Brucellosis incidence grew exponentially and spread to all 32 provinces. We describe the magnitude and the etiological distribution changes in mainland China by genotyping data and emphasize its recent reemergence. We also provide the genetic diversity and molecular epidemiological characteristics of Brucella.

Results: From a total of 206 Brucella isolates, 19 MLST genotypes (STs) were identified and 13 new STs(ST71-83) were found. MLST grouped the population into three clusters. B. melitensis, B. abortus and B. suis were grouped into cluster 1, 2 and 3 respectively. The predominant genotype in the first cluster by MLST, remained unchanged during the three stages. However, the proportion of genotypes in the three stages had changed. More isolates were clustered in ST8 at the re-emergence stage. STs71-74, which were not found in the two former stages, appeared at the re-emergence stage.
\end{abstract}

Conclusions: The changing molecular epidemiology of brucellosis improve our understanding of apparent geographic expansion from the historically affected north of China to southern provinces in recent reemergence.

Keywords: Brucella, Molecular epidemiology, Genotype, MLST

\section{Background}

Human brucellosis remains one of the most common zoonosis that occurs around the world [1] and is endemic in most areas of the world such as the Middle East, Western Asia, Africa, and South America [2]. The prevalence of human brucellosis in China has significantly increased in the past decades. The reported annual human brucellosis incidence showed a fast increasing tendency, from 0.07 per 100, 000 in 1990 to 3.33 per 100, 000 in 2013 [3]. The endemic situation of brucellosis in China has undergone three different stages [4]. It was highly endemic during mid-1950s and 1970s, which subsequently decreased until

\footnotetext{
* Correspondence: jianghai@icdc.cn

${ }^{\dagger}$ Equal contributors

${ }^{1}$ State Key Laboratory for Infectious Disease Prevention and Control,

Collaborative Innovation Center for Diagnosis and Treatment of Infectious Diseases, National Institute for Communicable Disease Control and Prevention, Chinese Center for Disease Control and Prevention, Beijing, China

Full list of author information is available at the end of the article
}

the mid-1990s, and then markedly increased to date [5]. In the past decades, Brucella multiple-locus variable number tandem repeat analysis (MLVA) and multilocus sequencing typing (MLST) had been proposed as a complementary technical approach to classical biotyping methods [6-8], based on its high capacity to identify specific genotypes [9] and establish important epidemiological information, assist in tracing the origin of brucellosis outbreak [10], investigate the genetic relationship within a strain group, and discriminate atypical strains among biovars and species [11].

B. melitensis is the predominant species that has been associated with human outbreaks and sporadic brucellosis cases in China, and B. abortus and B. suis have also been associated with sporadic epidemics [5]. A previous study has shown that the MLST genotype of Brucella in Inner Mongolia has undergone significant changes that could be depicted as three stages. In China, The MLST method identified 18 known ST types: ST7,ST8,ST34,ST35 
and ST37 (B. melitensis biovar 1 and 3), ST1, ST2, ST5, ST28, ST29, ST30, ST31, ST32, ST33 and ST38(B. abortus biovar 1 and 3), and ST14, ST17 and ST36 (B. suis biovar 1and 3) [3, 12-14]. During the past decades, outbreaks of human brucellosis have been reported in increasing numbers and with an apparent geographic expansion from the historically affected north of Chinato southern provinces [15]. To investigate etiological changes of brucellosis in China, species, biovars and genotypes of Brucella isolates were comprehensively analyzed and compared.

\section{Methods}

\section{Bacterial strains and DNA preparation}

A total of 206 Brucella isolates, including 158 B. melitensis (118 from human, 21 from sheep, 7 from cattle, 2 from yak, 1 from camel, 1 from deer, 1 from dog, and 7 unknown), 26 B. abortus (12 from cattle, 7 from human, 4 from sheep, 2 from yak, and 1 unknown), and $22 B$. suis (12 from human, 5 from pig, 1 from deer, 1 from goat, 1 from sheep, and 2 unknown), were collected from human and various animals in 25 provinces between 1953 and 2013 (Additional file 1: Table S1). All isolates were selected from the strain base of the culture collection center of the State Key Laboratory for Infectious Disease Prevention and Control, National Institute for Communicable Disease Control and Prevention, Chinese Center for Disease Control and Prevention, which serves as a repository of strains isolated from other Centers for Disease Control and Prevention around the country.

All isolates were cultured to stationary phase at $37{ }^{\circ} \mathrm{C}$ in Brucella broth. Brucella strains were identified as Brucella species and biovars according to the traditional biochemical reaction [16], including phage lysis test with $\mathrm{Tb}, \mathrm{BK} 2$, and $\mathrm{R}$ phages, and agglutination with monospecific $A$ and $M$ antisera. Whole genomic DNA was extracted from Brucella cultures using a DNeasy Blood and Tissue Kit (Qiagen China Ltd., China) following the manufacturer's protocol for extraction of genomic DNA from gram-negative bacteria. All sample handling was performed in a BSL-3 biocontainment laboratory at the National Institute for Communicable Disease Control and Prevention, Chinese Center for Disease Control and Prevention(ICDC, China CDC).

\section{Scheme of MLST}

MLST was performed using the method described previously. [7] 9 distinct genomic loci were selected, including seven housekeeping genes, one outer membrane protein gene and one intergenic fragment. PCR cycling parameters were as follows: $95^{\circ} \mathrm{C}$ for $5 \mathrm{~min}$, followed by 30 cycles of $94{ }^{\circ} \mathrm{C}$ for $30 \mathrm{~s}, 63^{\circ} \mathrm{C}$ for $30 \mathrm{~s}, 72{ }^{\circ} \mathrm{C}$ for $60 \mathrm{~s}$, and an elongation step at $72{ }^{\circ} \mathrm{C}$ for $10 \mathrm{~min}$. The resultant PCR products were purified and sequenced at
Shengong Bioscience Company(Shanghai, China). The sequence data were edited using EditSeq module of the Lasergene package (version 7).

\section{Data analysis of MLST}

Each allele of the nine loci was given a distinct numerical designation according to previously published MLST database $[6,7]$. Each unique allelic profile for the nine loci was identified as a sequence type (ST).Cluster analysis was performed with UPGMA using the software BioNumerics version 5.1(Applied-Maths, Inc.). A minimum spanning tree (MST) was constructed to determine the minimum evolution path from one strain to all others on the network with a categorical coefficient (with 1/HGDI weight) using the BioNumerics software version 5.1 (Applied-Maths, Inc.). The maps showing the distribution of genotypes in China were drawn with ArcGIS 10.2 for Desktop. Fisher's exact test was performed with SAS $9.3[17,18]$.

\section{Results \\ Different prominent species and biovars during three incidence stages}

As shown in Additional file 1: Table S1, in 1950-1960 s, 11 B. abortus (7 biovar 1,1 biovar 2 and 3 biovar 3), 17 B. melitensis (6 biovar 1, 7 biovar 2 and 4 biovar 3 ) and 7 B. suis biovar 1 were collected. And in 1970-1980s, 11 B. abortus (2 biovar 1, 1 biovar 3,2 biovar 6,1 biovar 7 and 5 biovar 9), 23 B. melitensis (15 biovar 1, 5 biovar 2 and 3 biovar 3 ) and 10 B. suis (7 biovar 1, 1 biovar 2 and 2 biovar 3)were collected. While in 1990-2000 s, 4 B. abortus (2biovar 1and 2biovar 3), 118 B. melitensis (17 biovar 1 and 106 biovar 3) and 5 B. suis biovar 3were collected. During three incidence stages the most common species was always $B$. melitensis. But, $B$. melitensis biovar 1 was popular before $1990 \mathrm{~s}$ and B. melitensis biovar 3 was predominant in recent re-emergence.

\section{Genetic diversity of 206 Brucella isolates using MLST analysis}

ST (9 loci) provided a discriminatory power of 0.565 (Simpson index). Of the 9 loci, aroA was the most discriminatory (Simpson index: 0.481) (the Simpson index of all loci ranged from 0.230 to 0.481 ). (Table 1 ).

B. melitensis, B. abortus and B. suis were grouped into cluster 1,2 and 3 respectively. Nineteen distinct STs were identified among the 9 loci of MLST. Thirteen new STs that were found in the present study were designated as ST71-83. These new STs sequences data were given in Additional file 2: File S2. Among each cluster, the predominant ST in China was ST8 $(n=137)$, ST2 $(n=13)$ and ST17 $(n=7)$, respectively. For ST8, there were extensive hosts, including sheep, goat, cattle, deer, yak, camel and human. For ST2, 
Table 1 Polymorphism indexes of ST loci in the 206 Brucella isolates

\begin{tabular}{lllll}
\hline Locus & Diversity index & Number of types & Max $_{(\mathrm{a} i)^{\mathrm{b}}}$ \\
\hline ST (9 loci) & 0.565 & $05 \%$ Confidence interval & 19 & 0.651 \\
aroA & 0.481 & $0.485-0.644$ & 8 & 0.703 \\
omp25 & 0.450 & $0.405-0.558$ & 6 & 0.722 \\
cobQ & 0.436 & $0.364-0.508$ & 4 & 0.726 \\
glk & 0.413 & $0.337-0.489$ & 4 & 0.726 \\
gap & 0.406 & $0.333-0.479$ & 3 & 0.750 \\
int-hyp & 0.401 & $0.331-0.472$ & 3 & 0.750 \\
trpE & 0.388 & $0.323-0.453$ & 4 & 0.750 \\
dnaK & 0.308 & $0.234-0.382$ & 4 & 0.821 \\
gyrB & 0.230 & $0.158-0.303$ & 3 & 0.873 \\
\hline Simpson & & &
\end{tabular}

${ }^{a}$ Simpson index

${ }^{b}$ Fraction of samples with the highest frequency in each particular locus (range: $0.0-1.0$ )

there were 4 hosts, including cattle, sheep, yak and human. For ST17, there were only 2 hosts, pig and human (Additional file 1: Table S1). In recent reemergence, there were some changes among each cluster. In cluster 1 , some new STs were also found as follows: ST72, ST73, ST74 and ST75. In cluster 2, $B$. abortus biovar was more limited than before. In cluster 3, B. suis biovar 3 was predominant and ST17 strains were isolated from Guangxi in1962 as well as 52 years later in Hainan.

There is a good consistency between the genotype identified by MLST and the species determined by traditional typing methods. B. melitensis biovar 1 and 3 comprised isolates with genotypes $7,8,71,72,73,74,75$, and 81. B. abortus biovar 1 and 3 consisted of isolates with genotypes $1,2,5,79$, and 82 . B. suis biovar 1 and 3 included isolates with genotypes $17,76,77,78,80$, and 83 (Table 2 and Additional file 1: Table S1).

As B. melitensis is the main epidemic pathogenic species in China, its isolates were compared to those from provincial level. ST8 was the most widely distributed genotype. Inner Mongolia and Xinjiang remained the most variable provinces based on MLST results. Furthermore, Sichuan, Guangdong, and Guangxi also showed several STs. Genotypic overlaps are presented in Additional file 1: Table S1 (Fig. 1).

\section{Comparison of STs changes at three incidence stages}

MLST analysis showed that in each of the 3 stages, cluster 1, which comprised all B. melitensis isolates, remained as the main cluster, whereas relatively fewer strains belonged to clusters 2 and 3(Additional file 3: Figure S1 and Table 2). In addition, a higher number of strains were clustered into cluster 1, particularly in genotype ST8, which was concurrent with an epidemic. ST7275 , which were not detected in the former two stages, were detected at the re-emergence stage (Table 2). The changes in the proportion of isolates in the three clusters among various incidence stages were also statistically significant $(P=3.885 \mathrm{E}-11, n=206)$.

\section{Discussion}

Human brucellosis has reemerged in mainland China since the mid-1990s and has expanded geographically from northern to southern China. Previous epidemiological data

Table 2 ST distributions during three endemic stages

\begin{tabular}{|c|c|c|c|c|}
\hline \multirow[t]{2}{*}{ Cluster } & \multirow[t]{2}{*}{ STs } & \multicolumn{3}{|c|}{ Incidence stage ${ }^{a}$} \\
\hline & & $\begin{array}{l}\text { High endemic } \\
\text { 1950-1960s }\end{array}$ & $\begin{array}{l}\text { Decline } \\
\text { 1970-1980s }\end{array}$ & $\begin{array}{l}\text { Re-emergence } \\
\text { 1990-2010s }\end{array}$ \\
\hline \multirow[t]{8}{*}{ S1 } & 7 & 0 & 1 & 4 \\
\hline & 8 & 17 & 20 & 100 \\
\hline & 71 & 0 & 2 & 0 \\
\hline & 72 & 0 & 0 & 1 \\
\hline & 73 & 0 & 0 & 1 \\
\hline & 74 & 0 & 0 & 10 \\
\hline & 75 & 0 & 0 & 1 \\
\hline & 81 & 0 & 1 & 0 \\
\hline \multirow[t]{5}{*}{ S2 } & 1 & 3 & 3 & 1 \\
\hline & 2 & 5 & 5 & 3 \\
\hline & 5 & 3 & 1 & 0 \\
\hline & 79 & 1 & 0 & 0 \\
\hline & 82 & 0 & 1 & 0 \\
\hline \multirow[t]{6}{*}{ S3 } & 17 & 2 & 0 & 5 \\
\hline & 76 & 1 & 3 & 0 \\
\hline & 77 & 0 & 5 & 0 \\
\hline & 78 & 3 & 1 & 0 \\
\hline & 80 & 1 & 0 & 0 \\
\hline & 83 & 0 & 1 & 0 \\
\hline
\end{tabular}

a The number represents the amount of strains in a particular ST in each respective incidence stage 


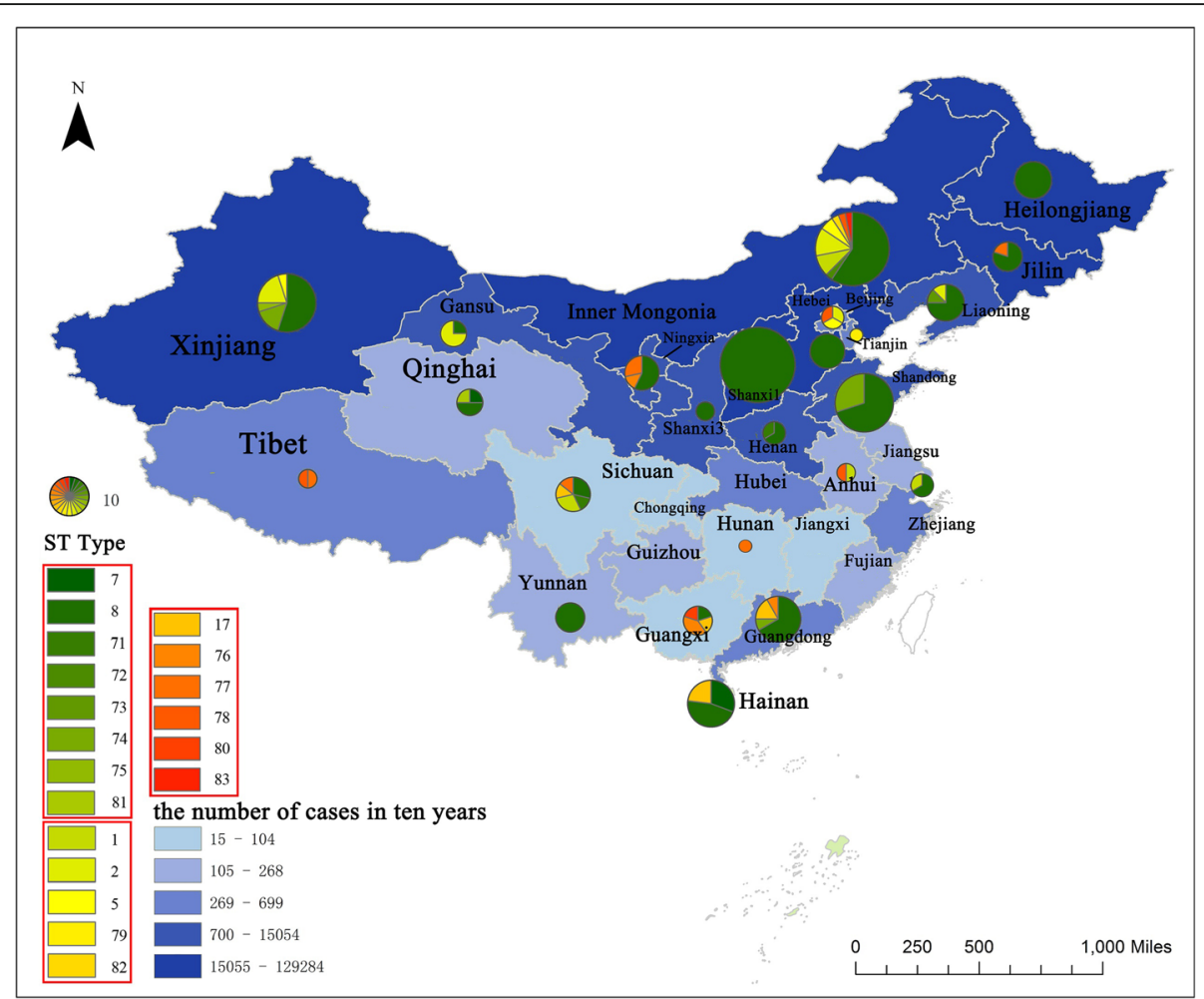

Fig. 1 Distribution of MLST in China. Size of circles reflects the number of isolates in a particular province. Color of sectors reflects the genotypes of isolates as the legend displays. The genotypes belong to one identical cluster were framed with red line in the legend. Shade of blocks reflects the number of cases in recent ten years in a particular province

showed that B. melitensis was predominant species that was associated with outbreaks throughout the country [16]. Results from the present study further implied that different biovars of this species were associated with the two high incidence stages. However, no distinct relationship between biovar and genotyping was observed. A previous study also determined that neither MLVA nor MLST can effectively discriminate $B$. melitensis biovars [19]. These findings indicate that variable number tandem-repeat loci and single-nucleotide polymorphisms, which provide congruent data, might have independently evolved putative genetic determinants in these biovars.

In history, brucellosis was first reported in Inner Mongolia [20] and then eventually spread from the north to the south, and now has now been reported in all 32 Chinese provinces [21]. As seen in both maps, the number of cases in a span of 10 years was highest in the north and gradually declined in the south. Provinces with the highest number of cases, Inner Mongolia and Xinjiang, contained the most variable genotypes that were composed of $B$. melitensis, $B$. abortus, and B. suis (Fig. 1). The newly-emerging provinces such Hainan and re-emerging provinces [22] such as Guangdong, also consisted of several genotypes that were even not less than the old epidemic area in the north. The prevention and control of brucellosis in China thus appears to be a very challenging task. $B$. melitensis and $B$. suis were the main causative pathogens of the cases reported in Hainan. Guangxi, which is located adjacent to Hainan, is an important epidemic area of $B$. suis in China. A previous report indicated that Hainan farmers tend to import piglets from Guangxi [23]. In the present study, the strains isolated from Hainan were clustered together with those from Guangxi and showed the same genotype, ST17. Due to insufficiency of epidemiological information, the pigs scattered in the backyard that drink from the same water source as humans, sick pigs, and abortuses might be haphazardly disposed [24]. The importation of infected animals and the limited measure in brucellosis prevention might be the reasons that have caused further spread of brucellosis among pigs and human beings [23]. B. melitensis in Hainan, which was isolated in 2013 and genotyped as ST7, were clustered together with the strains from Qinghai that were isolated in 1986 , with a similarity of about $90 \%$ by MLST. Qinghai province is in the northwest region and Hainan in the very south region of China. The origin of these strains remains unclear. B. melitensis and B. suis were the main causative pathogens for cases reported in Guangdong. However, these cases were mainly detected in urban areas [25]. Human migration from the north to the south, increased livestock trading and meat production consumption, lack of livestock 
quarantine measures, and unsafe eating habits [15, 25] might be the main factors that contributed to human infection, without that need for direct contact with livestock.

The appearance of STs 72-74 at the re-emergence stage (isolated in 2011 and 2012 from Inner Mongolia, Liaoning, Guangdong, Shandong, and Xinjiang provinces, which are all border provinces), might have induced the transfer of these 4 STs across the border, thereby resulting in its further spread and suggesting the urgent need to establish inspection and quarantine measures against further spread. At the two high incidence stages, STs were different and more STs (ST8, STs 7275 ) were founded at the re-emergence stage. The emergence of new genotypes of $B$. melitensis might be a reason for brucellosis incidence changes in China.

In this study, the phenomenon of host shift was common between $B$. melitensis and B. abortus, especially $B$. melitensis (sheep) and the accessory hosts (cattle, deer, yak, and camel). The results have highlighted some of the potential difficulty in national control programs. On the other hand, alterations in socioeconomic and political systems, increasing animal trade and a decreasing awareness by practitioners and public health authorities led to the reemergence of new endemic foci.

This study has some limitations. Due to insufficiencies in reporting brucellosis cases to the local CDC and the low positive rate of culture, there may be discrepancies among collected strains in the culture collection center as well as in its prevalence, thereby causing detection bias. Because brucellosis involves an extensive duration period, a more precise description of the distribution of prevalent genotypes in China should be the focus of future research studies.

\section{Conclusions}

MLST could be used for the epidemiological surveillance of brucellosis. The changing molecular epidemiology of brucellosis improve our understanding of apparent geographic expansion from the historically affected north of China to southern provinces in recent reemergence.

\section{Additional files}

Additional file 1: Table S1. Origins and genotyping results of 206 Brucella strains using MLST analysis. (Also see Figure S1 for column information. (XLS $46 \mathrm{~kb}$ )

Additional file 2: File S2. New STs (ST71-ST83) sequences data. (DOCX $37 \mathrm{~kb})$

Additional file 3: Figure S1. UPGMA dendrogram based on the MLST assay showing the similarities of 206 Brucella isolates. Key: serial number for the 206 isolates; Biovar: stains species and biovars by phenotype; Place/Time: the place and time when the strains were collected; Source: the hosts from which the bacteria was isolated; ST: MLST genotype. (PDF $221 \mathrm{~kb}$ )

\section{Abbreviations}

CE: Capillary electrophoresis; MST: Minimum spanning tree; STs: MLST genotypes; UPGMA: Unweighted pair group method using arithmetic averages

\section{Acknowledgements \\ Not applicable.}

\section{Funding}

This study was funded by the National Nature Science Foundation (81271900) and National Key Program for Infectious Diseases of China (2013ZX10004805-005). The funders contributed to the study design and data collection.

\section{Availability of data and materials}

The data in this study were extracted from the National Notifiable Disease surveillance System (http://chinacdc.cn/wps/portal/).

\section{Authors' contributions}

HJ, BYC and WXF conceived and designed the study. DRP, XL, DDD performed MLVA typing. DRP also drafted the manuscript. DDD, PX and XL performed the MLVA cluster analysis and manuscript revision. ZZZ, LQX, GZT and HYZ prepared the DNA samples. JH critically reviewed the manuscript All authors read and approved the final manuscript.

\section{Ethics approval and consent to participate}

This research was carried out according to the principles of the Declaration of Helsinki [26].This study is a retrospective investigation of historical collections strains using modern typing methods and study protocol was approved by the Ethics Committees of National Institute for Communicable Disease Control and Prevention, Chinese Center for Disease Control and Prevention. Informed consent was obtained from all the patients before diagnosis in the study. We isolated Brucella. spp. from patients' blood samples with their agreement.

\section{Consent for publication}

Not applicable.

\section{Competing interests}

The authors declare that they have no competing interests.

\section{Publisher's Note}

Springer Nature remains neutral with regard to jurisdictional claims in published maps and institutional affiliations.

\section{Author details}

${ }^{1}$ State Key Laboratory for Infectious Disease Prevention and Control, Collaborative Innovation Center for Diagnosis and Treatment of Infectious Diseases, National Institute for Communicable Disease Control and Prevention, Chinese Center for Disease Control and Prevention, Beijing, China. ${ }^{2}$ Laboratory of Zoonoses, China Animal Health and Epidemiology Center, MOA, Qingdao, China. ${ }^{3}$ National Institute of Occupational Health and Poison Control, Chinese Center for Disease Control and Prevention, Beijing, China. ${ }^{4}$ Qinghai Institute for Endemic Disease Prevention and Control, Xining, China

Received: 20 June 2017 Accepted: 3 January 2018

Published online: 10 January 2018

\section{References}

1. Corbel MJ. Brucellosis: an overview. Emerg Infect Dis. 1997;3(2):213-21.

2. Pappas G, Papadimitriou P, Akritidis N, Christou L, Tsianos EV. The new global map of human brucellosis. Lancet Infect Dis. 2006;6(2):91-9.

3. Lai S, Zhou H, Xiong W, Gilbert M, Huang Z, Yu J, Yin W, Wang L, Chen Q, Li Y, et al. Changing epidemiology of human brucellosis, China, 1955-2014. Emerg Infect Dis. 2017;23(2):184-94.

4. Cui B. Surveillance and control of brucellosis in China. Disease Surveillance. 2007;10:649-51.

5. Deqiu S, Donglou X, Jiming Y. Epidemiology and control of brucellosis in China. Vet Microbiol. 2002;90(1-4):165-82. 
6. Sankarasubramanian J, Vishnu US, Khader LK, Sridhar J, Gunasekaran P. Rajendhran: BrucellaBase: genome information resource. Infect Genet Evol. 2016;43:38-42.

7. Whatmore AM, Perrett LL, MacMillan AP. Characterisation of the genetic diversity of Brucella by multilocus sequencing. BMC Microbiol. 2007;7:34.

8. Maiden MC, Bygraves JA, Feil E, Morelli G, Russell JE, Urwin R, Zhang Q, Zhou J, Zurth K, Caugant DA, et al. Multilocus sequence typing: a portable approach to the identification of clones within populations of pathogenic microorganisms. Proc Natl Acad Sci U S A. 1998;95(6):3140-5.

9. Jiang $H$, Wang $H, X u L, H u G, M a J$, Xiao P, Fan W, Di D, Tian G, Fan M, et al. MLVA genotyping of Brucella melitensis and Brucella abortus isolates from different animal species and humans and identification of Brucella suis vaccine strain S2 from cattle in China. PLoS One. 2013;8(10):e76332.

10. Massis FD, Ancora M, Atzeni M, Rolesu S, Bandino E, Danzetta ML, Zilli K, Giannatale ED, Scacchia M. MLVA as an epidemiological tool to trace back Brucella melitensis Biovar 1 re-emergence in Italy. Transboundary \& Emerging Diseases. 2015;62(5):463-9.

11. Maio E, Begeman L, Bisselink Y, van Tulden P, Wiersma L, Hiemstra S, Ruuls R, Grone A, Roest HI, Willemsen P, et al. Identification and typing of Brucella spp. in stranded harbour porpoises (Phocoena Phocoena) on the Dutch coast. Vet Microbiol. 2014;173(1-2):118-24.

12. Chen Y, Ke Y, Wang Y, Yuan X, Zhou X, Jiang H, Wang Z, Zhen Q, Yu Y, Huang $L$, et al. Changes of predominant species/biovars and sequence types of Brucella isolates, Inner Mongolia, China. BMC Infect Dis. 2013;13:514

13. Ma JY, Wang $H$, Zhang XF, LQ X, GY H, Jiang $H$, Zhao F, Zhao HY, Piao DR, Qin YM, et al. MLVA and MLST typing of Brucella from Qinghai, China. Infect Dis Poverty. 2016:5:26.

14. Sun MJ, Di DD, Li Y, Zhang ZC, Yan H, Tian LL, Jing ZG, Li JP, Jiang H, Fan WX. Genotyping of Brucella melitensis and Brucella abortus strains currently circulating in Xinjiang, China. Infect Genet Evol. 2016;44:522-9.

15. Jiang H, Fan M, Chen J, Mi J, Yu R, Zhao H, Piao D, Ke C, Deng X, Tian G, et al. MLVA genotyping of Chinese human Brucella melitensis biovar 1,2 and 3 isolates. BMC Microbiol. 2011;11:256.

16. Alton $\mathrm{GG}$, Jones $\mathrm{LM}$, Pietz DE. Laboratory techniques in brucellosis. Monogr Ser World Health Organ. 1975;55:1-163.

17. Ormsby T: Getting to know ArcGIS desktop, 2nd edn. Redlands, Calif.: ESRI Press; 2010.

18. SAS Institute. SAS 9.3 intelligence platform : overview. Cary, N.C: SAS Institute Inc.; 2011

19. Kattar MM, Jaafar RF, Araj GF, Le Fleche P, Matar GM, Abi Rached R, Khalife $S$, Vergnaud $G$. Evaluation of a multilocus variable-number tandem-repeat analysis scheme for typing human Brucella isolates in a region of brucellosis endemicity. J Clin Microbiol. 2008:46(12):3935-40.

20. Zia SH, Wang FL. Brucellosis in north China; a clinical, etiological and epidemiological study. The American journal of tropical medicine and hygiene. 1949;29(6):925-36.

21. Li YJ, Li XL, Liang S, Fang LQ, Cao WC. Epidemiological features and risk factors associated with the spatial and temporal distribution of human brucellosis in China. BMC Infect Dis. 2013;13:547.

22. Liu Q, Cao L, Zhu XQ. Major emerging and re-emerging zoonoses in China: a matter of global health and socioeconomic development for 1.3 billion. International journal of infectious diseases : IJID : official publication of the International Society for Infectious Diseases. 2014;25:65-72.

23. Weng $Y$, Yan $X$. Analysis on the epidemiologic characteristics of four cases of brucellosis. Hainan Medical Journal. 2013;24(17):2626-7.

24. Di D, Cui B, Wang H, Zhao H, Piao D, Tian L, Tian G, Kang J, Mao X, Zhang $X$, et al. Genetic polymorphism characteristics of Brucella canis isolated in China. PLoS One. 2014;9(1):e84862.

25. Jiang H, Du P, Zhang W, Wang H, Zhao H, Piao D, Tian G, Chen C, Cui B. Comparative genomic analysis of Brucella melitensis vaccine strain M5 provides insights into virulence attenuation. PLoS One. 2013;8(8):e70852.

26. WMA. Declaration of Helsinki - Ethical Principles for Medical Research Involving Human Subjects.2013.

\section{Submit your next manuscript to BioMed Central and we will help you at every step:}

- We accept pre-submission inquiries

- Our selector tool helps you to find the most relevant journal

- We provide round the clock customer support

- Convenient online submission

- Thorough peer review

- Inclusion in PubMed and all major indexing services

- Maximum visibility for your research

Submit your manuscript at www.biomedcentral.com/submit

) Biomed Central 